Documentation et bibliothèques

DOCUMENTATION BIBLIOTHËQUES

\title{
Mémoires de maîtrise soutenus à l'Ecole de bibliothéconomie de l’Université de Montréal depuis 1974
}

Volume 25, numéro 1, mars 1979

URI : https://id.erudit.org/iderudit/1054370ar

DOI : https://doi.org/10.7202/1054370ar

Aller au sommaire du numéro

\section{Éditeur(s)}

Association pour l'avancement des sciences et des techniques de la documentation (ASTED)

\section{ISSN}

0315-2340 (imprimé)

2291-8949 (numérique)

Découvrir la revue

Citer ce document

(1979). Mémoires de maîtrise soutenus à l'Ecole de bibliothéconomie de l'Université de Montréal depuis 1974. Documentation et bibliothèques, 25(1), 43-47. https://doi.org/10.7202/1054370ar

Tous droits réservés (C) Association pour l'avancement des sciences et des techniques de la documentation (ASTED), 1979
Ce document est protégé par la loi sur le droit d'auteur. L'utilisation des services d'Érudit (y compris la reproduction) est assujettie à sa politique d'utilisation que vous pouvez consulter en ligne.

https://apropos.erudit.org/fr/usagers/politique-dutilisation/ 


\section{chronique delarecherche}

\section{Mémoires de maîtrise soutenus à l'Ecole de bibliothéconomie de l'Université de Montréal depuis 1974}

Le programme de maîtrise en bibliothéconomie à l'Université de Montréal permet à l'étudiant de prendre l'option "recherche» et de rédiger un mémoire qui soit un apport original à la discipline. Depuis les débuts de la décennie 1970, peu d'étudiants se sont prévalus de cette possibilité ${ }^{1}$. Nous vous fournissons ici les sommaires de ces mémoires tels que rédigés par les étudiants eux-mêmes.

\section{Blain-Juneau, Jocelyne}

Les bibliotechniciens: leur formation et leur rôle dans les bibliothèques. Montréal, Université de Montréal, Ecole de bibliothéconomie, 1976. $219 \mathrm{p}$.

Des départements de Library Technology et de bibliotechnique ont formé, depuis une quinzaine d'années, des aides qualifiés pour seconder les bibliothécaires. Ce sont les library technicians aux Etats-Unis et au Canada anglais, les bibliotechniciens au Québec.

1. Pour les mémoires antérieurs à 1974, voir Georges Cartier, «L'Ecole de bibliothéconomie et la recherche», Documentation et bibliothèques, vol. 19, no 4 (décembre 1973), 174-175.
Ces départements ont multiplié les diplômés à un rythme tel que le marché du travail ne peut pas les absorber tous à la fois et ce, partout en Amérique. Plusieurs raisons sont à la base de cette anomalie qui semble plus cruciale au Québec qu'ailleurs.

Dans les bibliothèques, on a fait un partage des tâches techniques à confier à ces techniciens, suivant leur formation reçue au Cegep, afin de pouvoir les intégrer entre le professionnel et le commis. Plusieurs organigrammes de bibliothèque leur réservent des postes à combler, dès que les budgets permettront leur embauchage...

Les associations professionnelles de bibliothécaires et de bibliotechniciens essaient de leur venir en aide, mais les débouchés sur le marché du travail sont trop restreints pour le nombre toujours croissant de nouveaux diplômés.

L'auteur essaie de trouver les raisons de cette situation et de voir comment remédier à cette situation pénible dans laquelle s'engouffrent des jeunes gens pleins d'enthousiasme, qui pensent y trouver facilement un travail rémunérateur. II se demande, de plus, s'il ne faudra pas 
substituer la qualité à la quantité et fermer des écoles pour régulariser une telle situation?

\section{Bonville, Jean de}

Sources d'information et habitudes documentaires des journalistes de la presse quotidienne francophone $d u$ Québec. Montréal, Université de Montréal, École de bibliothéconomie, 1977. 215 p. ${ }^{2}$

Cette recherche a pour but d'identifier et d'analyser les sources d'information documentaire qu'utilisent, dans le cadre de leur activité professionnelle, les journalistes de la presse quotidienne francophone du Québec.

Dans un premier chapitre, à caractère méthodologique, l'auteur identifie son échantillon pour en tirer le profil sociodémographique. Puis, à partir de statistiques recueillies lors d'entrevues avec les journalistes, il tente de mesurer la part du temps de travail consacré à la documentation dans la semaine du journaliste.

Le chapitre II porte spécifiquement sur les sources d'information documentaire des journalistes. Après un aperçu des conditions de leur utilisation viennent l'énumération et l'évolution de ces sources d'information et leur évaluation. Suit une tentative d'interprétation des données recueillies lors de l'observation.

L'étude de la documentation personnelle des journalistes et des centres de documentation des journaux occupe le troisième chapitre.

Enfin, des considérations prospectives sur le rôle de la presse, du journaliste et de la documentation constituent le dernier chapitre.

2. Le mémoire de Jean de Bonville a été publié sous le titre, Le journaliste et sa documentation; sources d'information et habitudes documentaires des journalistes de la presse quotidienne francophone, Québec, Edi-GRIC, 1977, 236 p. Un résumé a paru dans cette revue: "Les habitudes documentaires des journalistes québécois». Documentation et bibliothèques, vol. 24 , no 1 (mars 1978), 33-35.

\section{Deguy-Lepage, Françoise}

Les débuts de la presse enfantine au Québec, L'Oiseau Bleu (1921-1940).

Montréal, Université de Montréal, Ecole de bibliothéconomie, $1977.133 \mathrm{p.}^{3}$

Ce mémoire constitue une analyse de contenu de la première revue québécoise pour la jeunesse, L'Oiseau Bleu, publiée par la Société Saint-Jean-Baptiste de Montréal, de 1921 à 1940.

L'analyse se divise en trois grandes parties, suivant en cela les étapes habituelles de tout processus de communication. La première partie a pour objet l'émetteur du message. Elle tente d'éclairer les raisons qui ont poussé la Société Saint-Jean-Baptiste à créer L'Oiseau Bleu, à une époque peu favorable à la publication, et retrace l'historique de la revue.

La deuxième partie étudie le message véhiculé par la revue. Le but de L'Oiseau Bleu étant avant tout didactique, un premier chapitre analyse les idées et les méthodes pédagogiques mises en oeuvre, tandis qu'un deuxième chapitre aborde les éléments qui font de L'Oiseau Bleu une revue spécifiquement destinée aux enfants: les jeux, la littérature adaptée à leur âge, les illustrations.

La troisième partie s'attache à découvrir les caractéristiques du récepteur du message: l'enfant et, inséparable de lui, sa famille. Enfin, des annexes et des index viennent compléter cette analyse.

\section{Demers, Jacques}

Etude sur la gestion des documents audiovisuels dans les bibliothèques des Cegeps francophones du Québec.

Montréal, Université de Montréal, Ecole de bibliothéconomie, 1975. 301 p. $^{4}$

Cette étude sur la gestion des documents audiovisuels dans les cegeps

3. Voir la synthèse de ce mémoire dans Documentation et bibliothèques; vol. 24, no 1 (mars 1978), 26-31.

4. Ce mémoire fut publié par l'ASTED, en 1977 , 306 p., dans la collection «Publications de l'Ecole de bibliothéconomie, no 6". 
francophones du Québec touche !es aspects suivants: structures administratives, sélection et acquisition, organisation technique, référence, prêt, promotion, locaux, collection, personnel, budget. Elle est basée sur une enquête menée auprès des collèges concernés et sur l'examen de la documentation professionnelle.

Des progrès intéressants ont été accomplis dans l'organisation des non-livres depuis la création des premiers collèges publics. Toutefois, on observe une différence notable entre la réalité et les conceptions présentées dans les ouvrages relatifs aux normes. Beaucoup de travail reste à faire pour transformer les bibliothèques de nos cegeps en centres documentaires multimédia regroupés à l'intérieur de centres de ressources éducatives. Les problèmes observés sont de plusieurs ordres: flottement dans les structures administratives, instruments de travail beaucoup moins satisfaisants dans le domáine de l'audiovisuel que dans l'imprimé, locaux inadéquats, doublage dans les chaînes de travail, formation inadéquate du personnel en place, collections encore très modestes, manque de collaboration entre les collèges.

Quelques priorités semblent se dégager pour l'avenir: rationalisation des structures administratives, perfectionnement du personnel, normalisation des procédures pour une meilleure utilisation des services centralisés, régionalisation des ressources documentaires.

\section{Fontaine, Jean-Marc}

Du collège-bibliothèque au cours-bibliothèque. Montréal, Université de Montréal, Ecole de bibliothéconomie, 1977. 197 p.

L'objet de ce mémoire est d'exposer la théorie et l'expérimentation d'une formule pédagogique, le cours-bibliothèque, basée sur la recherche documentaire de l'étudiant en bibliothèque.

La première partie explore l'histoire du concept de collège-bibliothèque tel qu'il s'est développé aux Etats-Unis, depuis les années 1960 principalement, sous l'impulsion de Louis Shores. Elle expose les caractéristiques de cette pédagogie et fait ressortir le rôle éminent que la bibliothèque $y$ joue.

La deuxième partie s'intéresse à l'intégration québécoise du concept de collège-bibliothèque. Elle analyse les principaux écrits relatifs à cette question et deux projets d'implantation de collège-bibliothèque au Québec.

La troisième partie présente les principales lignes d'un cours de philosophie basé sur les éléments du collège-bibliothèque. Grâce à un système qui mise sur l'engagement de l'étudiant dans une démarche individualisée et axée sur la recherche documentaire, ce cours vise à développer l'autonomie intellectuelle de l'étudiant et sa capacité de s'évaluer. Le chapitre rend compte de l'expérience et analyse les résultats des divers moyens de contrôle utilisés.

\section{Gallichan, Gilles}

Bibliothèques et culture au Canada après la conquête (1760-1800). Montréal, Université de Montréal, Ecole de bibliothéconomie, 1975. 145 p. $^{5}$

Cette recherche constitue une approche socioculturelle de l'histoire des bibliothèques au Québec à la fin du XVIIIe siècle. Aux lendemains de la Conquête, le Canada a connu une crise de l'éducation provoquée par la désorganisation du clergé sur qui reposait l'infrastructure de l'éducation.

De plus, dès les premières années du Régime anglais, les Canadiens ont récupéré rapidement mais superficiellement des institutions de tradition britannique comme les bibliothèques commerciales et les bibliothèques publiques. La bibliothèque fondée en 1779 par le Gouverneur Haldimand visait une intégration des Canadiens lettrés à la pensée britannique. Enthousiastes au départ, les Canadiens se retirèrent graduellement de ces bibliothèques qui finirent par n'être fréquentées que par les Britanniques.

5. Une synthèse a paru sous le même titre dans Georges-A. Chartrand, éd. Livre, bibliothèque et culture québécoise. Mélanges offerts à Edmond Desrochers, s.j., Montréal, ASTED, I: 299-310. 
C'est au niveau de leurs propres bibliothèques, celles des institutions et des collections privées, que l'on aperçoit mieux l'attitude des Canadiens face aux livres. On y constate une imperméabilité aux livres anglais pendant le premier demi-siècle de la domination britannique. L'étude des bibliothèques du XVIIIe siècle nous éclaire sur la genèse des institutions culturelles du Québec où toute une génération d'hommes publics canadiens ont puisé la matière de leur réflexion intellectuelle.

\section{Maltais (Hébert), Frances W.}

La bibliothèque dans la pédagogie du programme-cadre de français. Montréal, Université de Montréal, Ecole de bibliothéconomie, 1974. $95 \mathrm{p}$.

Le présent mémoire compare deux types de bibliothèques scolaires en rapport avec le programme-cadre de français au secondaire. Un élément de ce programme, l'étude des thèmes, requiert l'utilisation de ressources regroupées dans un centre de documentation ou dans une bibliothèque centralisée.

Par une recherche expérimentale, l'auteur tente de mesurer l'efficacité respective de ces deux types de bibliothèques dans l'apprentissage du travail intellectuel. L'expérience comportait trois groupes distincts de niveau secondaire I d'une centaine d'élèves chacun. L'un deux (groupe A) n'a utilisé que le centre de documentation, un autre (groupe $C$ ) que la bibliothèque, et un troisième (groupe $B$ ) avait accès aux deux. Le contrôle a été fait au moyen de questionnaires, d'exercices en bibliothèque et d'analyse des fiches de lecteur.

Les résultats des trois groupes sont relativement semblables. Cependant, le groupe $\mathrm{C}$, qui représente un milieu social légèrement moins favorisé, accuse une certaine faiblesse par rapport aux deux autres, sauf sur un point, la connaissance de la classification décimale. Notre expérience démontre que d'autres facteurs que le type de bibliothèque ont, dans l'acquisition de connaissances relatives aux instruments du travail intellectuel, une influence prépondérante, en particulier la méthode pédagogique utilisée par les professeurs.

\section{Olivier, Daniel}

Philéas Gagnon, bibliophile. Montréal, Université de Montréal, Ecole de bibliothéconomie, 1978. $96 \mathrm{p}$.

Cette étude veut éclairer la vie et surtout la carrière bibliophilique du québécois Philéas Gagnon en mettant à jour les principales méthodes d'accroissement de collection auxquelles il a eu recours.

Né en 1854 dans le faubourg Saint-Roch à Québec, Gagnon s'y établit, vingt ans plus tard, après de brèves études, comme marchand-tailleur. Ses débuts en affaires coïncident avec ses premières activités bibliophiliques. Se restreignant au départ au marché local, c'est-à-dire aux librairies de Québec ainsi qu'aux multiples ventes aux enchères qui se tenaient couramment dans cette ville, Gagnon acquit rapidement les connaissances de base et les relations qui allaient lui permettre de développer une bibliothèque de canadiana remarquable. Vers 1884 , Gagnon élargit ses réseaux d'acquisition. II établit des contacts avec des bibliophiles et libraires des Etats-Unis et de l'Europe au moyen de revues et des annuaires spécialisés en bibliophilie. II recevait régulièrement d'innombrables catalogues qu'il dépouillait systématiquement, toujours à l'affût d'aubaines et de raretés. II commenca aussi à publier ses propres catalogues de vente: entre 1884 et 1910 défilèrent dans les pages de ses catalogues autant de pièces qu'en contenait sa collection.

En 1895, Gagnon publia le catalogue raisonné de sa bibliothèque: l'Essai. Bien qu'il fût déjà passablement connu des spécialistes, ce fut la révélation à l'extérieur de ce cercle. D'un peu partout au Canada et aux Etats-Unis, de nombreux membres de l'élite intellectuelle eurent recours à ses services et à ses conseils.

Nommé en 1898 conservateur des Archives judiciaires du district de Québec, puis protonotaire, Gagnon continua son oeuvre jusqu'à ce qu'une santé défaillante 
et le besoin de fournir une certaine sécurité matérielle à sa famille ne l'amènent à se résigner à la vente de sa collection. En 1910, par l'entremise de l'abbé Nazaire Dubois, la ville de Montréal devenait propriétaire de la collection.

Par son activité, Gagnon contribua, au même titre que certains de ses contemporains tels Verreau, Sicotte, Dubois, Baby et autres, à nous assurer qu'une partie importante de notre héritage bibliophilique demeure intacte, à la disposition des chercheurs d'ici.

\section{Thibodeau-Brunet, Lise}

Réflexion sur les méthodes de construction de thesaurus à la lumière d'une expérience: l'élaboration d'un lexique de l'éducation spéciale. Montréal, Université de Montréal, Ecole de bibliothéconomie, 1975. $116 \mathrm{p}$.

Le thesaurus est un outil de plus en plus utilisé pour l'analyse et la recherche documentaires. Divers types de thesaurus existent selon que l'on considère la clientèle à qui ils sont destinés et leur structure particulière. Ces caractéristiques sont des facteurs importants dont il faut tenir compte au moment de l'élaboration d'un thesaurus.

Bien que le thesaurus soit un ouvrage d'un genre relativement nouveau, il existe une certaine tradition en ce qui concerne les méthodes pour l'élaborer. C'est pourquoi la fabrication d'un micro-thesaurus multilingue et international sur l'éducation spéciale s'appuyant sur une méthodologie différente de celle qui est généralement connue est devenue une expérience intéressante au niveau du développement des méthodes. Cette expérience a consisté à établir d'abord un lexique avec traduction et définition des termes avant de passer à leur structuration.

Une analyse comparative des méthodes courantes de construction de thesaurus et de la méthode utilisée pour l'établissement du lexique de l'éducation spéciale a permis de vérifier l'intérêt de cette dernière méthode pour certains thesaurus aux caractéristiques particulières.

\title{
PUBLICATIONS ROGER SECRETT LTÉE
}

\section{Offre}

a) Un Département des archives disposant de plus d'un million d'exemplaires de journaux et de périodiques en sciences humaines, venant de tous les pays du monde.

b) Un Service d'abonnement qui aide le bibliothécaire à tenir à jour, et dans la limite de son budget, le Département des périodiques.

c) Un Atelier de reliure, soit pour réparer toutes vos collections défraîchies, soit pour relier, à des prix très raisonnables, vos nouvelles acquisitions selon vos spécifications. Des informations sont disponibles sur simple demande écrite.

Tous nos clients de langue française sont priés d'adresser leurs demandes de renseignements à:

\author{
Mademoiselle Lorraine Laliberté \\ Directrice de la Section française \\ Publications Roger Secrett Ltée \\ 120, chemin Midwest, Unité \#3 \\ SCARBOROUGH (Ontario) M1P 3B2
}

Tél.: (416) 751-0599 